\title{
AMERICAN PRICES EMBEDDED IN EUROPEAN PRICES
}

\author{
Benjamin JOURDAIN $^{\mathrm{a}}$, Claude MARTINI ${ }^{\mathrm{b}}$ \\ a ENPC-CERMICS, 6-8 av. Blaise-Pascal, Cité Descartes, Champs-sur-Marne, \\ 77455 Marne la Vallée cedex 2, France \\ b INRIA Projet Mathfi, Domaine de Voluceau, Rocquencourt, BP105, \\ 78153 Le Chesnay cedex, France
}

Received 12 January 2000

ABSTRACT. - In this paper, we are interested in American option prices in the Black-Scholes model. For a large class of payoffs, we show that in the region where the European price increases with the time to maturity, this price is equal to the American price of another claim. We give examples in which we explicit the corresponding claims. The characterization of the American claims obtained in this way remains an open question.

(C) 2001 L'Association Publications de l'Institut Henri Poincaré. Published by Elsevier B.V. All rights reserved

Keywords: Optimal stopping, Free boundary problems, Martingales, Black-Scholes model, European options, American options

AMS classification: 60G40, 60G46, 90A09

RÉSUMÉ. - Ce travail met en évidence un lien entre prix d'options européennes et prix d'options américaines dans le modèle de Black-Scholes. Nous montrons que pour une large classe de fonctions de payoff $\varphi$, dans la zone où il augmente avec la maturité, le prix de l'option européenne est égal au prix américain correspondant à une fonction de payoff modifiée $\widehat{\varphi}$. Nous donnons des exemples où il est possible d'expliciter $\widehat{\varphi}$. Mais la caractérisation de l'image de $\varphi \rightarrow \widehat{\varphi}$ reste un problème ouvert.

(C) 2001 L'Association Publications de l'Institut Henri Poincaré. Published by Elsevier B.V. All rights reserved

\section{Introduction}

Consider the classical Black-Scholes model:

$$
\begin{gathered}
d X_{t}^{x}=\rho X_{t}^{x} d t+\sigma X_{t}^{x} d B_{t}, \\
X_{0}^{x}=x>0, \\
\rho \in \mathbb{R}, \sigma>0,
\end{gathered}
$$

E-mail addresses: jourdain@cermics.enpc.fr (B. Jourdain), Claude.Martini@inria.fr (C. Martini). 
where $B$ is a standard Brownian motion, $\rho$ the instantaneous interest rate and $\sigma$ the volatility of $X$ and denote by

$$
\mathcal{A} f(x)=\frac{\sigma^{2} x^{2}}{2} f^{\prime \prime}(x)+\rho x f^{\prime}(x)-\rho f(x)
$$

the Black-Scholes infinitesimal generator. Given a continuous function $\psi: \mathbb{R}_{+}^{*} \rightarrow \mathbb{R}_{+}$ satisfying some growth assumptions, the price of the so-called American option with payoff $\psi$, maturity $t>0$ and spot $x$ is given by the expression

$$
v_{\psi}^{a m}(t, x)=\sup _{\tau \in \mathcal{T}(0, t)} \mathbb{E}\left[\mathrm{e}^{-\rho \tau} \psi\left(X_{\tau}^{x}\right)\right]
$$

where $\tau$ runs across the set of stopping times of the Brownian filtration such that $\tau \leqslant t$ almost surely. Except for some very particular class of payoffs $\psi$ (e.g. payoffs satisfying $\forall x>0, \mathcal{A} \psi(x) \geqslant 0$ or $\forall x>0, \mathcal{A} \psi(x) \leqslant 0)$, in general, there is no closedform expressions for $v_{\psi}^{a m}(t, x)$. The computation of $v_{\psi}^{a m}(t, x)$ usually relies either on finite-difference type methods or Markov-chain approximation methods to solve the corresponding optimal stopping problem in a discrete time-space framework. There is also a huge literature on special approximation methods designed for some particular payoffs, among which the case of the Put option, given by $\psi(x)=(K-x)^{+}$where $K$ is some positive constant (the strike of the option) has received much attention.

The purpose of this paper is to exhibit a new class of payoffs $\psi$ for which a closed-form expression for $v_{\psi}^{a m}(t, x)$ is available. The idea originates from the analytic properties of the function $v_{\psi}^{a m}$ : this function is greater than $\psi$ by $(0.2)$ and typically the space $] 0, \infty\left[\times \mathbb{R}_{+}^{*}\right.$ splits into two regions, the so-called Exercise region where by definition $v_{\psi}^{a m}=\psi$ and its complement the Continuation region where $v_{\psi}^{a m}>\psi$. It is known that $v_{\psi}^{a m}$ solves the evolution equation associated with $(0.1)$

$$
\partial_{t} v_{\psi}^{a m}=\mathcal{A} v_{\psi}^{a m}
$$

in the Continuation region (at least in the distribution sense). Moreover, as from (0.2) $t \mapsto v_{\psi}^{a m}(t, x)$ is non-decreasing, $\partial_{t} v_{\psi}^{a m} \geqslant 0$ holds. In fact, since $v_{\psi}^{a m}$ is a continuous function, it may be remarked that the knowledge of $v_{\psi}^{a m}$ in the Continuation region is enough to get $v_{\psi}^{a m}$ everywhere.

This leads to the natural idea to build American prices (i.e. functions $v_{\psi}^{a m}$ for some $\psi$ ) by picking up the classical solution $v_{\varphi}(t, x)$ of the evolution equation:

$$
\left\{\begin{array}{l}
\forall t, x>0, \partial_{t} v_{\varphi}(t, x)=\mathcal{A} v_{\varphi}(t, x), \\
\forall x>0, v_{\varphi}(0, x)=\varphi(x)
\end{array}\right.
$$

in the region where it increases with time. From a financial point of view, $v_{\varphi}(t, x)$ is the Black-Scholes price of the European option with payoff $\varphi$ and maturity $t$ i.e. $v_{\varphi}(t, x)=\mathbb{E}\left[\mathrm{e}^{-\rho t} \varphi\left(X_{t}^{x}\right)\right]$. This embedding idea has been worked out in [2] in case $\rho=0$. A similar approach has also been developped in the different context of the free boundary arising in a two-phases problem (see [1]). Trying to generalize things to the 
case $\rho \neq 0$, we ran across a probabilistic proof which allows a very compact statement of the embedding result.

The first section of the paper is devoted to some basic properties of European and American prices within the Black-Scholes model. Next we state and prove our embedding theorem (Section 2). Then we give some examples (Section 3). Lastly, we discuss some properties of the map which takes a payoff $\varphi$ to the payoff $\widehat{\varphi}$ the American price of which is embedded in its European price (Section 4). The characterization of the payoffs $\widehat{\varphi}$ obtained in this way remains an open question.

\section{European and American prices in the Black-Scholes model}

In this section we recall the very few properties of European and American BlackScholes prices we shall need in the next section.

Let $\alpha=2 \rho / \sigma^{2}$. The invariant functions of the semigroup associated with $(0.1)$ are easily seen to be the vector space generated by $x$ and $x^{-\alpha}$. We shall consider payoffs $\varphi$ such that

$$
x \in \mathbb{R}_{+}^{*} \mapsto \varphi(x) \in \mathbb{R}_{+} \text {is continuous and } \sup _{x>0} \frac{\varphi(x)}{x+x^{-\alpha}}<\infty
$$

The growth assumption is only there to grant the existence of the various expectations involved. It seems that the continuity assumption could be removed, but the connection with American options would be more intricate, so we keep this hypothesis.

PROPOSITION 1 ([3]). - Under (H0) the price of the European option with maturity $t \geqslant 0$ and payoff $\varphi$ is given by

$$
v_{\varphi}(t, x)=\mathbb{E}\left[\mathrm{e}^{-\rho t} \varphi\left(X_{t}^{x}\right)\right] .
$$

In particular $v_{\varphi}(0, x)=\varphi(x)$.

The function $v_{\varphi}$ is continuous from $\left[0, \infty\left[\times \mathbb{R}_{+}^{*}\right.\right.$ into $\mathbb{R}$, and for any $t>0$, the process $\left(\mathrm{e}^{-\rho u} v_{\varphi}\left(t-u, X_{u}^{x}\right)\right)_{0 \leqslant u \leqslant t}$ is a continuous square-integrable martingale.

Last, for $\phi(y)=\varphi\left(\mathrm{e}^{y}\right) /\left(\mathrm{e}^{y}+\mathrm{e}^{-\alpha y}\right)$,

$$
v_{\varphi}(t, x)-x \mathbb{E}\left(\phi\left(\sigma B_{t}+\left(\rho+\frac{\sigma^{2}}{2}\right) t\right)\right)-x^{-\alpha} \mathbb{E}\left(\phi\left(\sigma B_{t}-\left(\rho+\frac{\sigma^{2}}{2}\right) t\right)\right)
$$

converges to 0 as $t \rightarrow+\infty$ locally uniformly in $x>0$.

Proof. - We only prove the last assertion which is quite unusual. By definition of $\phi$,

$$
\begin{aligned}
v_{\varphi}(t, x)= & \mathbb{E}\left(\mathrm{e}^{-\rho t} X_{t}^{x} \phi\left(\ln (x)+\sigma B_{t}+\left(\rho-\frac{\sigma^{2}}{2}\right) t\right)\right) \\
& +\mathbb{E}\left(\mathrm{e}^{-\rho t}\left(X_{t}^{x}\right)^{-\alpha} \phi\left(\ln (x)+\sigma B_{t}+\left(\rho-\frac{\sigma^{2}}{2}\right) t\right)\right) .
\end{aligned}
$$

Since $\mathrm{e}^{-\rho t} X_{t}^{x}=x \mathrm{e}^{\sigma B_{t}-\frac{\sigma^{2}}{2} t}$ and $\mathrm{e}^{-\rho t}\left(X_{t}^{x}\right)^{-\alpha}=x^{-\alpha} \mathrm{e}^{-\frac{2 \rho}{\sigma} B_{t}-\frac{2 \rho^{2}}{\sigma^{2}} t}$, by Girsanov theorem, we deduce that 


$$
\begin{aligned}
v_{\varphi}(t, x)= & x \mathbb{E}\left(\phi\left(\ln (x)+\sigma B_{t}+\left(\rho+\frac{\sigma^{2}}{2}\right) t\right)\right) \\
& +x^{-\alpha} \mathbb{E}\left(\phi\left(\ln (x)+\sigma B_{t}-\left(\rho+\frac{\sigma^{2}}{2}\right) t\right)\right) .
\end{aligned}
$$

Now,

$$
\begin{aligned}
& \left|\mathbb{E}\left(\phi\left(\ln (x)+\sigma B_{t}+\left(\rho+\frac{\sigma^{2}}{2}\right) t\right)\right)-\mathbb{E}\left(\phi\left(\sigma B_{t}+\left(\rho+\frac{\sigma^{2}}{2}\right) t\right)\right)\right| \\
& \quad=\frac{1}{\sqrt{2 \pi}}\left|\int_{\mathbb{R}} \phi\left(\sigma \sqrt{t} y+\left(\rho+\frac{\sigma^{2}}{2}\right) t\right)\left(\mathrm{e}^{-\frac{(y-\ln (x) /(\sigma \sqrt{t}))^{2}}{2}}-\mathrm{e}^{-\frac{y^{2}}{2}}\right) d y\right| \\
& \leqslant \frac{1}{\sqrt{2 \pi}} \sup _{z>0} \frac{\varphi(z)}{z+z^{-\alpha}} \int_{\mathbb{R}}\left|\mathrm{e}^{-\frac{(y-\ln (x) /(\sigma \sqrt{t}))^{2}}{2}}-\mathrm{e}^{-\frac{y^{2}}{2}}\right| d y \\
& \leqslant\left(\mathrm{e}^{\frac{\ln ^{2}(x)}{2 \sigma^{2} t}}-1\right) \sup _{z>0} \frac{\varphi(z)}{z+z^{-\alpha}}
\end{aligned}
$$

converges to 0 as $t \rightarrow+\infty$ locally uniformly for $x>0$.

We deal with $\mathbb{E}\left(\phi\left(\ln (x)+\sigma B_{t}-\left(\rho+\sigma^{2} / 2\right) t\right)\right)$ in the same way to conclude.

Let us now turn to American options:

PROPOSITION 2 ([3]). - If $\psi$ satisfies (H0), the price of the American option with maturity $t \geqslant 0$ and payoff $\psi$ is given by

$$
v_{\psi}^{a m}(t, x)=\sup _{\tau \in \mathcal{T}(0, t)} \mathbb{E}\left[\mathrm{e}^{-\rho \tau} \psi\left(X_{\tau}^{x}\right)\right]
$$

where $\tau$ runs across the set of stopping times of the Brownian filtration such that $\tau \leqslant t$ almost surely. In particular $v_{\psi}^{a m}(0, x)=\psi(x)$.

The function $v_{\psi}^{a m}$ is continuous from $\left[0, \infty\left[\times \mathbb{R}_{+}^{*}\right.\right.$ into $\mathbb{R}$, and for any $x \in \mathbb{R}_{+}^{*}$ the map $t \mapsto v_{\psi}^{a m}(t, x)$ is non-decreasing.

\section{Embedding American prices in European prices}

Our main result relates the price $v_{\varphi}(t, x)$ of the European option with payoff $\varphi$ to the price $v_{\widehat{\varphi}}^{a m}(t, x)$ of the American option with payoff $\widehat{\varphi}(x)=\inf _{t \geqslant 0} v_{\varphi}(t, x)$ :

THEOREM 3. - Under (H0) let

$$
\widehat{\varphi}(x)=\inf _{t \geqslant 0} v_{\varphi}(t, x) .
$$

Then

$$
\forall(t, x) \in[0,+\infty) \times \mathbb{R}_{+}^{*}, \quad \sup _{\tau \in \mathcal{T}(0, t)} \mathbb{E}\left[\mathrm{e}^{-\rho \tau} \widehat{\varphi}\left(X_{\tau}^{x}\right)\right] \leqslant v_{\varphi}(t, x),
$$

where the supremum is taken over all the stopping times $\tau$ of the filtration of the Brownian motion smaller than $t$. 
Moreover, if there exists a continuous function $\widehat{t}: \mathbb{R}_{+}^{*} \rightarrow[0,+\infty]$ such that

$$
\forall x>0, \quad \inf _{t \geqslant 0} v_{\varphi}(t, x)=v_{\varphi}(\widehat{t}(x), x)
$$

(where $v_{\varphi}(\infty, x)$ is defined as $\left.\liminf _{t \rightarrow+\infty} v_{\varphi}(t, x)\right)$ then the converse inequality holds for $t \geqslant \widehat{t}(x)$ and

$$
\forall(t, x) \in[0,+\infty) \times \mathbb{R}_{+}^{*}, \quad \sup _{\tau \in \mathcal{T}(0, t)} \mathbb{E}\left[\mathrm{e}^{-\rho \tau} \widehat{\varphi}\left(X_{\tau}^{x}\right)\right]=v_{\varphi}(t \vee \widehat{t}(x), x) .
$$

If, lastly, either $\widehat{t}\left(x_{0}\right)<+\infty$ for some $x_{0}>0$, or

$$
\exists C>0, \forall x, y>0, \quad|\varphi(x)-\varphi(y)| \leqslant C\left(|x-y|+\left|x^{-\alpha}-y^{-\alpha}\right|\right)
$$

or the function $\frac{\varphi(x)}{x+x^{-\alpha}}$ admits limits both for $x \rightarrow 0$ and $x \rightarrow+\infty$, then the function $\hat{\varphi}$ satisfies $(\mathrm{H} 0)$ and

$$
\forall(t, x) \in[0,+\infty) \times \mathbb{R}_{+}^{*}, \quad v_{\widehat{\varphi}}^{a m}(t, x)=v_{\varphi}(t \vee \widehat{t}(x), x) .
$$

Proof. - Let $(t, x) \in \mathbb{R}_{+} \times \mathbb{R}_{+}^{*}$. According to Proposition 1, the process $\left(\mathrm{e}^{-\rho u} v_{\varphi}(t-\right.$ $\left.\left.u, X_{u}^{x}\right)\right)_{u \in[0, t]}$ is a martingale. If $\tau \leqslant t$ is a stopping time, by Doob optional sampling theorem

$$
v_{\varphi}(t, x)=\mathbb{E}\left[\mathrm{e}^{-\rho \tau} v_{\varphi}\left(t-\tau, X_{\tau}^{x}\right)\right] \geqslant \mathbb{E}\left[\mathrm{e}^{-\rho \tau} \widehat{\varphi}\left(X_{\tau}^{x}\right)\right] .
$$

Since $\tau$ is arbitrary, we deduce that (2.1) holds.

To prove (2.2), we suppose the existence of $\widehat{t}: \mathbb{R}_{+}^{*} \rightarrow[0,+\infty]$ continuous such that

$$
\forall x>0, \quad \widehat{\varphi}(x)=v_{\varphi}(\widehat{t}(x), x)
$$

and we make a distinction between the two following situations:

- Case $\widehat{t}(x)=+\infty$ : since $s \rightarrow \sup _{\tau \in \mathcal{T}(0, s)} \mathbb{E}\left[\mathrm{e}^{-\rho \tau} \widehat{\varphi}\left(X_{\tau}^{x}\right)\right]$ is increasing, for $u \geqslant t$

$$
\widehat{\varphi}(x) \leqslant \sup _{\tau \in \mathcal{T}(0, t)} \mathbb{E}\left[\mathrm{e}^{-\rho \tau} \widehat{\varphi}\left(X_{\tau}^{x}\right)\right] \leqslant \sup _{\tau \in \mathcal{T}(0, u)} \mathbb{E}\left[\mathrm{e}^{-\rho \tau} \widehat{\varphi}\left(X_{\tau}^{x}\right)\right] \leqslant v_{\varphi}(u, x) \quad \text { by }(2.1) .
$$

Letting $u \rightarrow+\infty$, we deduce that

$$
\forall t \geqslant 0, \quad \sup _{\tau \in \mathcal{T}(0, t)} \mathbb{E}\left[\mathrm{e}^{-\rho \tau} \widehat{\varphi}\left(X_{\tau}^{x}\right)\right]=\widehat{\varphi}(x)=v_{\varphi}(\infty, x)=v_{\varphi}(t \vee \widehat{t}(x), x) .
$$

- Case $\widehat{t}(x)<+\infty$ : let $t \geqslant \widehat{t}(x)$ and $\tau_{0}=\inf \left\{u: t-u-\widehat{t}\left(X_{u}^{x}\right) \leqslant 0\right\}$. Since $\widehat{t}\left(X_{t}^{x}\right) \geqslant 0$, the stopping time $\tau_{0}$ is smaller than $t$. By continuity of $u \rightarrow t-u-\widehat{t}\left(X_{u}^{x}\right)$, $t-\tau_{0}=\widehat{t}\left(X_{\tau_{0}}^{x}\right)$. Hence

$$
\begin{aligned}
v_{\varphi}(t, x) & =\mathbb{E}\left[\mathrm{e}^{-\rho \tau_{0}} v_{\varphi}\left(t-\tau_{0}, X_{\tau_{0}}^{x}\right)\right]=\mathbb{E}\left[\mathrm{e}^{-\rho \tau_{0}} v_{\varphi}\left(\widehat{t}\left(X_{\tau_{0}}^{x}\right), X_{\tau_{0}}^{x}\right)\right] \\
& =\mathbb{E}\left[\mathrm{e}^{-\rho \tau_{0}} \widehat{\varphi}\left(X_{\tau_{0}}^{x}\right)\right] \leqslant \sup _{\tau \in \mathcal{T}(0, t)} \mathbb{E}\left[\mathrm{e}^{-\rho \tau} \widehat{\varphi}\left(X_{\tau}^{x}\right)\right] .
\end{aligned}
$$


The converse inequality (2.1) is already proved. Hence $\forall t \geqslant \widehat{t}(x), \sup _{\tau \in \mathcal{T}(0, t)}$ $\mathbb{E}\left[\mathrm{e}^{-\rho \tau} \widehat{\varphi}\left(X_{\tau}^{x}\right)\right]=v_{\varphi}(t, x)$ and $\tau_{0}$ is an optimal stopping time. Since $t \rightarrow v_{\widehat{\varphi}}^{a m}(t, x)$ is increasing, for $t \leqslant \widehat{t}(x)$,

$$
\widehat{\varphi}(x) \leqslant \sup _{\tau \in \mathcal{T}(0, t)} \mathbb{E}\left[\mathrm{e}^{-\rho \tau} \widehat{\varphi}\left(X_{\tau}^{x}\right)\right] \leqslant \sup _{\tau \in \mathcal{T}(0, \widehat{t}(x))} \mathbb{E}\left[\mathrm{e}^{-\rho \tau} \widehat{\varphi}\left(X_{\tau}^{x}\right)\right]=\widehat{\varphi}(x),
$$

which concludes the proof of (2.2).

If we check that $\hat{\varphi}$ is continuous under the various assumptions made in the last assertion of the theorem then (2.3) follows immediately since $v_{\widehat{\varphi}}^{a m}(t, x)=\sup _{\tau \in \mathcal{T}(0, t)}$ $\mathbb{E}\left[\mathrm{e}^{-\rho \tau} \widehat{\varphi}\left(X_{\tau}^{x}\right)\right]$.

- If $\widehat{t}\left(x_{0}\right)<+\infty$ for some $x_{0}>0$ then for $x$ in a neighbourhood of $x_{0}, \widehat{t}(x)<+\infty$ and since $t \rightarrow \sup _{\tau \in \mathcal{T}(0, t)} \mathbb{E}\left[\mathrm{e}^{-\rho \tau} \hat{\varphi}\left(X_{\tau}^{x}\right)\right]$ is increasing, by $(2.2) v_{\varphi}(t, x)$ has a limit for $t \rightarrow+\infty$. By the last assertion of Proposition 1, we deduce that for $\phi(y)=$ $\varphi\left(\mathrm{e}^{y}\right) /\left(\mathrm{e}^{y}+\mathrm{e}^{-\alpha y}\right), \mathbb{E}\left(\phi\left(\sigma B_{t}+\left(\rho+\sigma^{2} / 2\right) t\right)\right)$ and $\mathbb{E}\left(\phi\left(\sigma B_{t}-\left(\rho+\sigma^{2} / 2\right) t\right)\right)$ admit limits as $t \rightarrow+\infty$. We denote the limits by $a$ and $b$. Proposition 1 then yields that $v_{\varphi}(t, x)$ converges to the invariant function $a x+b x^{-\alpha}$ as $t \rightarrow+\infty$ locally uniformly for $x>0$. The continuity of $\widehat{\varphi}$ follows easily.

- If $\varphi(x) /\left(x+x^{-\alpha}\right)$ admits limits for $x \rightarrow 0$ and $x \rightarrow+\infty$ then $\phi(y)$ admits limits for $y \rightarrow-\infty$ and $y \rightarrow+\infty$. We deduce that $\mathbb{E}\left(\phi\left(\sigma B_{t}+\left(\rho+\sigma^{2} / 2\right) t\right)\right)$ and $\mathbb{E}\left(\phi\left(\sigma B_{t}-\left(\rho+\sigma^{2} / 2\right) t\right)\right)$ admit limits as $t \rightarrow+\infty$ and we conclude like in the previous case.

- If $\forall x, y>0,|\varphi(x)-\varphi(y)| \leqslant C\left(|x-y|+\left|x^{-\alpha}-y^{-\alpha}\right|\right)$, then $\forall t \geqslant 0$,

$$
\begin{aligned}
\left|v_{\varphi}(t, x)-v_{\varphi}(t, y)\right| & \leqslant \mathbb{E}\left[\mathrm{e}^{-\rho t}\left|\varphi\left(X_{t}^{x}\right)-\varphi\left(X_{t}^{y}\right)\right|\right] \\
& \leqslant C\left(|x-y| \mathbb{E}\left[\mathrm{e}^{-\rho t} X_{t}^{1}\right]+\left|x^{-\alpha}-y^{-\alpha}\right| \mathbb{E}\left[\mathrm{e}^{-\rho t}\left(X_{t}^{1}\right)^{-\alpha}\right]\right) \\
& \leqslant C\left(|x-y|+\left|x^{-\alpha}-y^{-\alpha}\right|\right) .
\end{aligned}
$$

Hence the functions $x \rightarrow v_{\varphi}(t, x)$ indexed by $t \geqslant 0$ are equicontinuous, which ensures the continuity of $x \rightarrow \inf _{t \geqslant 0} v_{\varphi}(t, x)=\widehat{\varphi}(x)$.

Remark 4. - The continuity of the argument of the infimum is granted in the following uniqueness situation: suppose that $\forall x>0, \exists ! \widehat{t}(x) \leqslant T(x), \widehat{\varphi}(x)=v_{\varphi}(\widehat{t}(x), x)$ where $T: \mathbb{R}_{+}^{*} \rightarrow \mathbb{R}_{+}$is continuous. Then by the continuity of $T$ and $v_{\varphi}$, it is easy to see that $\widehat{\varphi}(x)=\inf _{t \in[0, T(x)]} v_{\varphi}(t, x)$ is continuous. Moreover, since $\widehat{t}(x)=\inf \{t \geqslant$ $\left.0: \hat{\varphi}(x)=v_{\varphi}(t, x)\right\}$ (respectively $\left.\widehat{t}(x)=\sup \left\{t \leqslant T(x): \widehat{\varphi}(x)=v_{\varphi}(t, x)\right\}\right), \widehat{t}$ is lower semi-continuous (respectively upper semi-continuous) i.e. $\widehat{t}$ is continuous and (2.3) holds.

In the above theorem it may happen that the function $\widehat{\varphi}$ is nil: in case $\lim _{x \rightarrow 0} \frac{\varphi(x)}{x+x^{-\alpha}}=$ $\lim _{x \rightarrow+\infty} \frac{\varphi(x)}{x+x^{-\alpha}}=0$, we easily check that

$$
\forall x>0, \quad \lim _{t \rightarrow+\infty} v_{\varphi}(t, x)=0 .
$$

In such a situation, the following localized version of our main result is far more interesting than Theorem 3. It is proved by the same arguments, after noticing that the 
continuity of $(t, x) \in[0,+\infty) \times \mathbb{R}_{+}^{*} \rightarrow v_{\varphi}(t, x)$ implies the continuity of $x \rightarrow \widehat{\varphi}^{T}(x)=$ $\inf _{0 \leqslant t \leqslant T} v_{\varphi}(t, x)$ where $T>0$.

THEOREM 5. - Let $T>0$. The function $\widehat{\varphi}^{T}(x)=\inf _{0 \leqslant t \leqslant T} v_{\varphi}(t, x)$ satisfies (H0) and

$$
\forall(t, x) \in[0, T] \times \mathbb{R}_{+}^{*}, \quad v_{\widehat{\varphi}^{T}}^{a m}(t, x) \leqslant v_{\varphi}(t, x)
$$

Moreover, if there exists a continuous function $\widehat{t}: \mathbb{R}_{+}^{*} \rightarrow[0, T]$ such that

$$
\forall x>0, \quad \inf _{0 \leqslant t \leqslant T} v_{\varphi}(t, x)=v_{\varphi}(\widehat{t}(x), x),
$$

then

$$
\forall(t, x) \in[0, T] \times \mathbb{R}_{+}^{*}, \quad v_{\widehat{\varphi}^{T}}^{a m}(t, x)=v_{\varphi}(t \vee \widehat{t}(x), x) .
$$

Remark 6. - The only feature of the Black-Scholes model which is required in the above results is time-homogeneity. In fact, Propositions 1 and 2 and Theorems 3 and 5 can be adapted to the so-called generalized Black-Scholes model:

$$
\begin{aligned}
& X_{t}^{x}=x \exp \left(\sigma B_{t}+\left(\rho-\delta-\frac{\sigma^{2}}{2}\right) t\right), \\
& v_{\varphi}(t, x)=\mathbb{E}\left[\mathrm{e}^{-\rho t} \varphi\left(X_{t}^{x}\right)\right] \quad \text { and } \\
& v_{\psi}^{a m}(t, x)=\sup _{\tau \in \mathcal{T}(0, t)} \mathbb{E}\left[\mathrm{e}^{-\rho \tau} \psi\left(X_{\tau}^{x}\right)\right],
\end{aligned}
$$

or to the more general time-homogeneous model:

$$
\begin{aligned}
& X_{0}^{x}=x, \quad d X_{t}^{x}=X_{t}^{x}\left(\sigma\left(X_{t}^{x}\right) d B_{t}+\left(\rho\left(X_{t}^{x}\right)-\delta\left(X_{t}^{x}\right)\right) d t\right) \\
& v_{\varphi}(t, x)=\mathbb{E}\left[\mathrm{e}^{-\int_{0}^{t} \rho\left(X_{s}^{x}\right) d s} \varphi\left(X_{t}^{x}\right)\right] \quad \text { and } \\
& v_{\psi}^{a m}(t, x)=\sup _{\tau \in \mathcal{T}(0, t)} \mathbb{E}\left[\mathrm{e}^{-\int_{0}^{\tau} \rho\left(X_{s}^{x}\right) d s} \psi\left(X_{\tau}^{x}\right)\right]
\end{aligned}
$$

and also to the multidimensional versions of these models.

Of course it would be of great interest to give conditions on $\varphi$ which ensure the existence of a continuous curve in the argument of the infimum. One way is to perform explicit computations, since the Black-Scholes semigroup is explicit. Nevertheless this is not very illuminating. We ran across the following statement, for the local embedding result, which is maybe the simplest in this direction:

PROPOSITION 7. - Let $\varphi$ be a $C^{4}$ function which satisfies (H0) and such that for some $x_{c} \in \mathbb{R}_{+}^{*}$,

(i) $\mathcal{A} \varphi\left(x_{c}\right)=0$ and either $\forall x>0,\left(x-x_{c}\right) \mathcal{A} \varphi(x) \geqslant 0$

$$
\text { or } \forall x>0,\left(x-x_{c}\right) \mathcal{A} \varphi(x) \leqslant 0 \text {. }
$$

(ii) $\mathcal{A}^{2} \varphi\left(x_{c}\right)>0$ and $\partial_{x} \mathcal{A} \varphi\left(x_{c}\right) \neq 0$.

Then there exists a constant $T>0$ such that the assumptions of Theorem 5 are satisfied. 
Proof. - Since $\varphi$ is $C^{4}$, the function $v_{\varphi}(t, x)$ belongs to $C^{2,4}\left(\mathbb{R}_{+} \times \mathbb{R}_{+}^{*}\right)\left(C^{2}\right.$ in $t$, $C^{4}$ in $x$ ) and satisfies the Black-Scholes partial differential equation $\partial_{t} v_{\varphi}=\mathcal{A} v_{\varphi}$ for $t \geqslant 0$ and not only $t>0$. Consider the equation $\partial_{t} v_{\varphi}(t, x)=0$ in a neighbourhood of $\left(0, x_{c}\right)$ in $\{(t, x), t \geqslant 0\}$. By derivation of the Black-Scholes evolution equation, $\partial_{t x}^{2} v_{\varphi}\left(0, x_{c}\right)=\partial_{x} \mathcal{A} \varphi\left(x_{c}\right) \neq 0$. Hence, by the implicit functions theorem, there is for some $\varepsilon>0$ a curve $\hat{x}$ :

$$
\widehat{x}:[0, \varepsilon] \rightarrow \mathbb{R}_{+}^{*}
$$

continuous on $[0, \varepsilon]$, with $\widehat{x}(0)=x_{c}$, such that $\partial_{t} v_{\varphi}(t, \widehat{x}(t))=0$, and $C^{1}$ on $] 0, \varepsilon$ [ with

$$
\partial_{t^{2}}^{2} v_{\varphi}(t, \widehat{x}(t))+\partial_{t x}^{2} v_{\varphi}(t, \widehat{x}(t)) \widehat{x}^{\prime}(t)=0 .
$$

Moreover by taking $\varepsilon$ small enough we can assume that $\widehat{x}^{\prime}(t)$ does not vanish and keeps the same sign as $\widehat{x}^{\prime}\left(0^{+}\right)=-\frac{\mathcal{A}^{2} \varphi\left(x_{c}\right)}{\partial_{x} \mathcal{A} \varphi\left(x_{c}\right)}$. We deduce that there exists a continuous function $\widehat{t}:\left[x_{c}, \widehat{x}(\varepsilon)\right] \rightarrow[0, \varepsilon]$ such that $\widehat{x}(\widehat{t}(x))=x$.

Assume $\widehat{x}^{\prime}\left(0^{+}\right)>0$. Then the function $\widehat{t}$ is increasing. Moreover, $\partial_{x} \mathcal{A} \varphi(x)<0$ which ensures $\forall x<x_{c}, \mathcal{A} \varphi(x) \geqslant 0$ and $\forall x>x_{c}, \mathcal{A} \varphi(x) \leqslant 0$. We set $T=\varepsilon$ and extend $\widehat{t}$ to $\mathbb{R}_{+}^{*}$ by setting $\widehat{t}(x)=T$ for $x>x_{c}+\varepsilon$ and $\widehat{t}(x)=0$ for $x<x_{c}$. The obtained function is continuous and the whole point is to show that for every $x$, the infimum of $t \mapsto v_{\varphi}(t, x)$ on $[0, T]$ is reached at $\widehat{t}(x)$. This amounts to show that $\partial_{t} v_{\varphi}(t, x)=\mathcal{A} v_{\varphi}(t, x)$ is nonpositive for $(t, x)$ above $\widehat{x}$ (i.e. for $t \leqslant T$ and $x \geqslant \hat{x}(t)$ ) and non-negative below. If $\left(P_{t}\right)_{t \geqslant 0}$ denotes the semigroup associated with $(0.1)$,

$$
\mathcal{A} v_{\varphi}(t, x)=\mathcal{A} P_{t} \varphi(x)=P_{t} \mathcal{A} \varphi(x) .
$$

Let $(t, x)$ belong to the above (respectively below) region. By the optimal stopping theorem, $\mathcal{A} v_{\varphi}(t, x)$ is equal to the expectation of the value of the martingale $\left(\mathrm{e}^{-\rho u} P_{t-u} \mathcal{A} \varphi\left(X_{u}^{x}\right)\right)_{0 \leqslant u \leqslant t}$ stopped at the border of the above (respectively below) region $\{(u, \widehat{x}(u)), u \in[0, \varepsilon]\} \cup\left\{(0, x), x \geqslant x_{c}\right\}$ (respectively $\{(u, \widehat{x}(u)), u \in[0, \varepsilon]\} \cup$ $\left.\left\{(0, x), x \leqslant x_{c}\right\}\right)$ which is non-positive (respectively non-negative) since $\left.\left.\forall t \in\right] 0, \varepsilon\right]$, $P_{u} \mathcal{A} \varphi(\widehat{x}(u))=\partial_{u} v_{\varphi}(u, \widehat{x}(u))=0$ and $\forall x \geqslant x_{c}, \mathcal{A} \varphi(x) \leqslant 0\left(\right.$ respectively $\forall x \geqslant x_{c}, \mathcal{A} \varphi(x)$ $\geqslant 0)$.

The case $\widehat{x}^{\prime}\left(0^{+}\right)<0$ is handled in the same way.

Example 8. - As an application, consider the family of payoffs

$$
\varphi_{a, b}(x)=x^{-\alpha}+x^{a}-x^{b},
$$

where $1>a>b>-\alpha$. Then for $x \geqslant 1, x^{a} \geqslant x^{b}$, for $x<1, x^{-\alpha}>x^{b}$ so that $\varphi_{a, b}$ is non-negative. Moreover

$$
\lim _{x \rightarrow 0} \frac{\varphi_{a, b}(x)}{x+x^{-\alpha}}=1, \quad \lim _{x \rightarrow+\infty} \frac{\varphi_{a, b}(x)}{x+x^{-\alpha}}=0
$$

and $\varphi_{a, b}$ satisfies $(\mathrm{H} 0)$. Let $\lambda(y)=\left(\frac{\sigma^{2}}{2} y+\rho\right)(y-1)$. Then

$$
\mathcal{A} \varphi_{a, b}(x)=\lambda(a) x^{a}-\lambda(b) x^{b},
$$


which gives, since $\lambda(a)<0$ and $\lambda(b)<0, \mathcal{A} \varphi_{a, b}(x)<0$ for $x>x_{c}$ and $\mathcal{A} \varphi_{a, b}(x)>0$ for $x<x_{c}$ with $\lambda(a) x_{c}^{a}=\lambda(b) x_{c}^{b}$. Moreover

$$
\mathcal{A}^{2} \varphi_{a, b}\left(x_{c}\right)=\lambda(a)^{2} x_{c}^{a}-\lambda(b)^{2} x_{c}^{b}=(\lambda(a)-\lambda(b)) \lambda(b) x_{c}^{b}
$$

and $\mathcal{A}^{2} \varphi_{a, b}\left(x_{c}\right)>0$ as soon as $\lambda(b)>\lambda(a)$. Lastly,

$$
\partial_{x} \mathcal{A} \varphi_{a, b}\left(x_{c}\right)=\lambda(a) a x_{c}^{a-1}-\lambda(b) b x_{c}^{b-1}=\left(\frac{a}{x_{c}}-\frac{b}{x_{c}}\right) \lambda(a) x_{c}^{a} \neq 0 .
$$

Of course, in this example, since $v_{\varphi}(t, x)=x^{-\alpha}+x^{a} \mathrm{e}^{t \lambda(a)}-x^{b} \mathrm{e}^{t \lambda(b)}$, everything can be computed explicitely and it is even possible to check the hypotheses of the global embedding result:

$$
\left(\frac{x \vee x_{c}}{x_{c}}\right)^{a-b}=\mathrm{e}^{\widehat{t}(x)(\lambda(b)-\lambda(a))}
$$

and

$$
\widehat{\varphi}_{a, b}(x)=x^{-\alpha}+x^{a}\left(\frac{x \vee x_{c}}{x_{c}}\right)^{\frac{\lambda(a)(a-b)}{\lambda(b)-\lambda(a)}}-x^{b}\left(\frac{x \vee x_{c}}{x_{c}}\right)^{\frac{\lambda(b)(a-b)}{\lambda(b)-\lambda(a)}} .
$$

Similarly the hypothesis of Proposition 7 are satisfied by the payoff $x+x^{b}-x^{a}$ where $1>a>b>-\alpha$ in case $\lambda(a)>\lambda(b)$.

In the global case, we could not find any simple condition on $\varphi$ ensuring the existence of a continuous curve in the argument of $\inf _{t \geqslant 0} v_{\varphi}(t, x)$. Nevertheless, it is worth mentioning the following interesting class of European payoffs: if $\varphi$ is a non-negative function equal to an invariant function $a x+b x^{-\alpha}$ with $a, b \geqslant 0, a+b>0$, less a nonnegative function $\phi$ satisfying $\lim _{x \rightarrow 0} \frac{\phi(x)}{x+x^{-\alpha}}=\lim _{x \rightarrow+\infty} \frac{\phi(x)}{x+x^{-\alpha}}=0$, then

$$
\begin{aligned}
& \forall x>0, \forall t \geqslant 0, \quad v_{\varphi}(t, x) \leqslant a x+b x^{-\alpha} \quad \text { and } \\
& \lim _{t \rightarrow+\infty} v_{\varphi}(t, x)=a x+b x^{-\alpha},
\end{aligned}
$$

which implies that $\widehat{\varphi}(x)=\inf _{t \geqslant 0} v_{\varphi}(t, x)$ is not trivial and that $\forall x \in \mathbb{R}, \exists \widehat{t}(x)<$ $+\infty, \widehat{\varphi}(x)=v_{\varphi}(\widehat{t}(x), x)$. The only assumption missing to apply Theorem 3 is the continuity of $\widehat{t}$.

The next section is dedicated to a family of payoffs $\varphi$ included in the above class. In these examples, we explicit some American prices with a non-trivial Exercise region thanks to Theorem 3. We also check that the above mentioned continuity of $\hat{t}$ is not always satisfied.

\section{Case study: $\alpha>-1$ and $\varphi(x)=x\left(1_{\left\{x<K_{1}\right\}}+1_{\left\{x>K_{2}\right\}}\right)$}

To be able to compare the invariant functions $x$ and $x^{-\alpha}$, we need to compare $-\alpha$ and 1. We choose the case $\alpha>-1$ which is the more interesting from a financial point of view since $\rho \geqslant 0 \Leftrightarrow \alpha \geqslant 0$. The payoff $\varphi$ is equal to the invariant function $x$ less the 
function $\phi(x)=x 1_{\left\{K_{1} \leqslant x \leqslant K_{2}\right\}}$. Since

$$
\forall x>0, \forall t>0, \quad 0<v_{\varphi}(t, x)<x \quad \text { and } \quad \lim _{t \rightarrow+\infty} v_{\varphi}(t, x)=x
$$

the function $t \rightarrow v_{\varphi}(t, x)$ is likely to be increasing for $K_{1}<x<K_{2}$ and decreasing then increasing otherwise. This remark together with the easiness of computations motivate the choice of this example. The function $\varphi$ satisfies the growth assumption in (H0) but is not continuous. Therefore, even if we make the computations for $\varphi$, we shall after all apply our results to a suitable regularization of $\varphi$.

\subsection{The case $K_{1}=0$}

To simplify notations, we replace $K_{2}$ by $K$ and write $\varphi(x)=x 1_{\{x>K\}}$. This payoff corresponds to the sum of one Call and $K$ Digit options with common strike $K$. Its simplicity allows to compute explicitely $\widehat{\varphi}$ and $\widehat{t}$.

Proposition 9. - Let $\varphi(x)=x 1_{\{x>K\}}$. Then

$$
v_{\varphi}(t, x)=x N\left(d_{1}(t, x)\right)
$$

where

$$
d_{1}(t, x)=\frac{\ln \left(\frac{x}{K}\right)+\left(\rho+\frac{\sigma^{2}}{2}\right) t}{\sigma \sqrt{t}}
$$

and $N(d)=\int_{-\infty}^{d} \mathrm{e}^{-\frac{y^{2}}{2}} \frac{d y}{\sqrt{2 \pi}}$ is the cumulative distribution function of the normal law. Moreover,

$$
\widehat{\varphi}(x)=x 1_{\{x>K\}} N\left(\frac{2}{\sigma} \sqrt{\left(\rho+\frac{\sigma^{2}}{2}\right) \ln \left(\frac{x}{K}\right)}\right)=v_{\varphi}(\widehat{t}(x), x)
$$

and

$$
\widehat{t}(x)=\frac{\ln (x / K) 1_{\{x \geqslant K\}}}{\rho+\frac{\sigma^{2}}{2}},
$$

and $\forall x>0, t \rightarrow v_{\varphi}(t, x)$ is strictly decreasing on $[0, \widehat{t}(x)]$ and strictly increasing on $[\widehat{t}(x),+\infty)$.

Proof. - Using Girsanov theorem, we get

$$
\begin{aligned}
v_{\varphi}(t, x) & =\mathbb{E}\left[x \mathrm{e}^{\sigma B_{t}-\frac{\sigma^{2}}{2} t} 1_{\left\{x e^{\sigma B_{t}+\left(\rho-\sigma^{2} / 2\right) t} \geqslant K\right\}}\right] \\
& =x P\left(x \mathrm{e}^{\sigma B_{t}+\left(\rho+\frac{\sigma^{2}}{2}\right) t} \geqslant K\right)=x N\left(d_{1}(t, x)\right) .
\end{aligned}
$$

By the chain rule, $\partial_{t} v_{\varphi}(t, x)=x N^{\prime}\left(d_{1}(t, x)\right) \partial_{t} d_{1}(t, x)$. Since $\forall x, t>0, x N^{\prime}\left(d_{1}(t, x)\right)>$ 0 and

$$
\partial_{t} d_{1}(t, x)=\frac{\left(\rho+\frac{\sigma^{2}}{2}\right) t-\ln \left(\frac{x}{K}\right)}{2 \sigma t^{3 / 2}}
$$


we obtain that

$$
\forall x>0, \quad\left\{\begin{array}{l}
\forall t \in] 0, \widehat{t}(x)\left[, \partial_{t} v_{\varphi}(t, x)<0\right. \\
\forall t>\widehat{t}(x), \partial_{t} v_{\varphi}(t, x)>0 .
\end{array}\right.
$$

Hence $\inf _{t \geqslant 0} v_{\varphi}(t, x)=v_{\varphi}(\widehat{t}(x), x)$ and the explicit expression of this function is easily computed.

Let us now regularize things in order to apply our theorem. Let $u>0$. The function $x \rightarrow v_{\varphi}(u, x)$ is continuous. Let $\left(P_{t}\right)_{t \geqslant 0}$ denote the semigroup associated with $(0.1)$. By the semigroup property, the price of the European option with payoff $v_{\varphi}(u, x)$ is $P_{t}\left(P_{u} \varphi\right)=P_{t+u} \varphi$. If we set $\widehat{\varphi}_{u}=\inf _{t \geqslant 0} P_{t}\left(P_{u} \varphi\right)$, then by the previous proposition, $\widehat{\varphi}_{u}(x)=v_{\varphi}(u \vee \widehat{t}(x), x)=P_{0 \vee \widehat{t}(x)-u)}\left(P_{u} \varphi\right)(x)$. Since $\widehat{t}$ is a continuous function with values in $[0,+\infty)$, so is $\widehat{t}_{u}(x)=0 \vee(\widehat{t}(x)-u)$. Applying Theorem 3 , we obtain the price of the American option with payoff $\widehat{\varphi}_{u}$ :

COROLlaRY 10. - Let $u>0$. The price of the American option with payoff $\widehat{\varphi}_{u}(x)=$ $v_{\varphi}(u \vee \widehat{t}(x), x)$ is

$$
\begin{aligned}
v_{\widehat{\varphi}_{u}^{a m}}^{a m}(t, x)= & v_{\varphi}((t+u) \vee \widehat{t}(x), x) \\
= & x\left(N \left(\frac{2}{\sigma} \sqrt{\left.\left(\rho+\frac{\sigma^{2}}{2}\right) \ln \left(\frac{x}{K}\right)\right)} 1_{\left\{t+u \leqslant \ln (x / K) /\left(\rho+\sigma^{2} / 2\right)\right\}}\right.\right. \\
& \left.+N\left(\frac{\ln \left(\frac{x}{K}\right)+\left(\rho+\frac{\sigma^{2}}{2}\right)(t+u)}{\sigma \sqrt{t+u}}\right) 1_{\left\{t+u>\ln (x / K) /\left(\rho+\sigma^{2} / 2\right)\right\}}\right)
\end{aligned}
$$

and the Exercise region is given by $\left\{(t, x): t+u \leqslant \ln (x / K) /\left(\rho+\sigma^{2} / 2\right)\right\}$.

Remark 11.-Although the payoff $\widehat{\varphi}_{u}$ has no financial meaning, this example provides a very interesting benchmark for numerical procedures devoted to American options since the price and the Exercise boundary are explicit. Let us also notice that this is a two-parameter ( $K$ and $u$ ) family of closed-formula. The payoff is of course obtained by setting $t$ to zero in $v_{\hat{\varphi}_{u}}^{a m}(t, x)$.

\subsection{The case $K_{1}>0$}

The main purpose of this subsection is to design an example where there is no continuous curve in the argument of the infimum (Proposition 13). By a slight modification of the computations made in the proof of Proposition 9, we get

$$
\begin{aligned}
& v_{\varphi}(t, x)=x\left(N\left(-d_{1}(t, x)\right)+N\left(d_{2}(t, x)\right)\right), \\
& \text { where for } i=1,2 d_{i}(t, x)=\frac{\ln \left(\frac{x}{K_{i}}\right)+\left(\rho+\frac{\sigma^{2}}{2}\right) t}{\sigma \sqrt{t}} .
\end{aligned}
$$

It is not possible to compute $\widehat{\varphi}$ explicitely but using the implicit functions theorem, we can study the sign of $\partial_{t} v_{\varphi}(t, x)$ to obtain:

LEMMA 12. - There exist two differentiable functions $t \in \mathbb{R}_{+}^{*} \rightarrow \xi_{1}(t)<\xi_{2}(t)$ satisfying 
(1) $\lim _{t \rightarrow 0} \xi_{i}(t)=K_{i}(i=1,2)$,

(2) $\forall t>0, \xi_{2}^{\prime}(t)>0$ and $\exists(\beta, T), 0<\beta<T \leqslant\left(1+\ln \sqrt{K_{2} / K_{1}}\right) /\left(2 \rho+\sigma^{2}\right)$, $\forall t<\beta, \xi_{1}^{\prime}(t)>0$ and $\forall t>T, \xi_{1}^{\prime}(t)<0$,

(3) $\forall t>0$,

$$
\xi_{2}(t)>K_{2} \mathrm{e}^{\left(\rho+\frac{\sigma^{2}}{2}\right) t} \quad \text { and } \quad \xi_{1}(t)<K_{1} \mathrm{e}^{\left(\rho+\frac{\sigma^{2}}{2}\right) t} \wedge \sqrt{K_{1} K_{2}} \mathrm{e}^{-\left(\rho+\frac{\sigma^{2}}{2}\right) t}
$$

and such that

$$
\begin{aligned}
& \forall t>0, \forall x \in] \xi_{1}(t), \xi_{2}(t)\left[, \quad \partial_{t} v_{\varphi}(t, x)>0 \quad\right. \text { and } \\
& \forall x \notin\left[\xi_{1}(t), \xi_{2}(t)\right], \quad \partial_{t} v_{\varphi}(t, x)<0 .
\end{aligned}
$$

Proof. - An easy computation yields that $\partial_{t} v_{\varphi}(t, x)$ is equal to the product of a strictly positive function with $f(t, \ln x)$ where

$$
\begin{aligned}
& f(t, y)=\left(y-a_{1}\right) \mathrm{e}^{b_{1} y+c_{1}}+\left(a_{2}-y\right) \mathrm{e}^{b_{2} y+c_{2}} \quad \text { where for } i=1,2, \\
& a_{i}(t)=\ln K_{i}+\left(\rho+\frac{\sigma^{2}}{2}\right) t, \quad b_{i}(t)=\frac{\ln K_{i}}{\sigma^{2} t}, \\
& c_{i}(t)=\left(\frac{\rho}{\sigma^{2}}+\frac{1}{2}\right) \ln K_{i}-\frac{\ln ^{2} K_{i}}{2 \sigma^{2} t} .
\end{aligned}
$$

Since $a_{1}<a_{2}, f\left(t, a_{2}\right)=\left(a_{2}-a_{1}\right) \mathrm{e}^{b_{1} a_{2}+c_{1}}>0$. Hence the function $y \rightarrow f(t, y)$ vanishes at the same points as

$$
y \rightarrow g(t, y)=\mathrm{e}^{\left(b_{2}-b_{1}\right) y+\left(c_{2}-c_{1}\right)}-\frac{y-a_{1}}{y-a_{2}} .
$$

As $a_{1}<a_{2}$ and $b_{1}<b_{2}$, the function $y \rightarrow g(t, y)$ is strictly increasing from -1 to $+\infty$ on ] $-\infty, a_{2}$ [ and from $-\infty$ to $+\infty$ on $] a_{2},+\infty$ [, so it vanishes exactly twice. Let $y_{1}<a_{2}<y_{2}$ denote the corresponding points. Since $\mathrm{e}^{\left(b_{2}-b_{1}\right) y_{1}+\left(c_{2}-c_{1}\right)}>0$ and $\frac{y_{1}-a_{1}}{y_{1}-a_{2}}<1$, we obtain respectively $y_{1}<a_{1}$ and $\left(b_{2}-b_{1}\right) y_{1}<c_{1}-c_{2}$. We combine these upperbounds to get

$$
y_{1}(t)<a_{1}(t) \wedge\left(\ln \sqrt{K_{1} K_{2}}-\left(\rho+\frac{\sigma^{2}}{2}\right) t\right) .
$$

We deduce that $x \rightarrow \partial_{t} v_{\varphi}(t, x)$ vanishes exactly twice, at the points $\xi_{1}(t)=\mathrm{e}^{y_{1}(t)}$ and $\xi_{2}(t)=\mathrm{e}^{y_{2}(t)}$ which satisfy statement (3). As $f\left(t, a_{2}\right)>0, \partial_{t} v_{\varphi}(t, x)$ is strictly positive for $x \in\left(\xi_{1}(t), \xi_{2}(t)\right)$. Moreover as $b_{1}<b_{2}, f(t, y)<0$ for $|y|$ large and $\partial_{t} v_{\varphi}(t, x)$ is strictly negative for $0<x<\xi_{1}(t)$ and for $x>\xi_{2}(t)$.

Let us study more precisely the functions $y_{1}(t)$ and $y_{2}(t)$. Since $\forall t>0, \forall y \neq$ $a_{2}(t), \partial_{y} g(t, y)>0$, by the implicit function theorem, for $i=1,2, y_{i}(t)$ is continuously differentiable and $y_{i}^{\prime}(t)$ has the same sign as $-\partial_{t} g\left(t, y_{i}(t)\right)$. Expliciting the dependence of $g$ on the time variable, we get

$$
g(t, y)=\exp \left(\frac{\ln \left(K_{2} / K_{1}\right)}{\sigma^{2} t}\left(y+\left(\rho+\frac{\sigma^{2}}{2}\right) t-\ln \sqrt{K_{1} K_{2}}\right)\right)-1
$$




$$
\begin{aligned}
& +\frac{\ln \left(K_{1} / K_{2}\right)}{y-\ln \left(K_{2}\right)-\left(\rho+\frac{\sigma^{2}}{2}\right) t}, \\
\partial_{t} g(t, y)= & \frac{\ln \left(K_{1} / K_{2}\right)}{\sigma^{2} t^{2}}\left(y-\ln \sqrt{K_{1} K_{2}}\right) \exp \left(\frac { \operatorname { l n } ( K _ { 2 } / K _ { 1 } ) } { \sigma ^ { 2 } t } \left(y+\left(\rho+\frac{\sigma^{2}}{2}\right) t\right.\right. \\
& \left.\left.-\ln \sqrt{K_{1} K_{2}}\right)\right)+\frac{\left(\rho+\frac{\sigma^{2}}{2}\right) \ln \left(K_{1} / K_{2}\right)}{\left(y-\ln K_{2}-\left(\rho+\frac{\sigma^{2}}{2}\right) t\right)^{2}} .
\end{aligned}
$$

Since $y_{2}(t)>a_{2}(t)>\ln \sqrt{K_{1} K_{2}}, \partial_{t} g\left(t, y_{2}(t)\right)$ is strictly negative and $\forall t>0, y_{2}^{\prime}(t)>0$. Moreover, when $t \rightarrow 0$ the first term in $g\left(t, y_{2}(t)\right)$ has a limit equal to $+\infty$ and the equation $g\left(t, y_{2}(t)\right)=0$ implies that the second term goes also to $\infty$ which gives $\lim _{t \rightarrow 0} y_{2}(t)=\ln K_{2}$.

By (3.1), $y_{1}(t)<a_{1}(t)=\ln K_{1}+\left(\rho+\sigma^{2} / 2\right) t$. Hence when $t \rightarrow 0$ the first term in $g\left(t, y_{1}(t)\right)$ has a limit equal to 0 . By considering the other terms we deduce that $\lim _{t \rightarrow 0} y_{1}(t)=\ln K_{1}$. Hence the first term in $\partial_{t} g\left(t, y_{1}(t)\right)$ goes to 0 and $\lim _{t \rightarrow 0} \partial_{t} g\left(t, y_{1}(t)\right)<0$. Therefore $\left.\exists \beta>0, \forall t \in\right] 0, \beta\left[, y_{1}^{\prime}(t)>0\right.$.

Using the equality $g\left(t, y_{1}(t)\right)=0$ to replace the exponential in $\partial_{t} g\left(t, y_{1}(t)\right)$ and multiplying by $\left(y_{1}(t)-\ln K_{2}-\left(\rho+\sigma^{2} / 2\right) t\right)^{2} / \ln \left(K_{2} / K_{1}\right)$, we obtain that $\partial_{t} g\left(t, y_{1}(t)\right)$ has the same sign as

$$
\begin{gathered}
\frac{-1}{\sigma^{2} t^{2}}\left(y_{1}(t)-\ln \sqrt{K_{1} K_{2}}\right)\left(y_{1}(t)-\ln K_{1}-\left(\rho+\frac{\sigma^{2}}{2}\right) t\right) \\
\times\left(y_{1}(t)-\ln K_{2}-\left(\rho+\frac{\sigma^{2}}{2}\right) t\right)-\left(\rho+\frac{\sigma^{2}}{2}\right) .
\end{gathered}
$$

As by (3.1), $y_{1}(t)<\ln \sqrt{K_{1} K_{2}}-\left(\rho+\sigma^{2} / 2\right) t$, we conclude that for some $T \leqslant$ $\left(1+\ln \sqrt{K_{2} / K_{1}}\right) /\left(2 \rho+\sigma^{2}\right), \forall t>T, \partial_{t} g\left(t, y_{1}(t)\right)>0$ and $y_{1}^{\prime}(t)<0$.

So the situation looks like in Fig. 1.

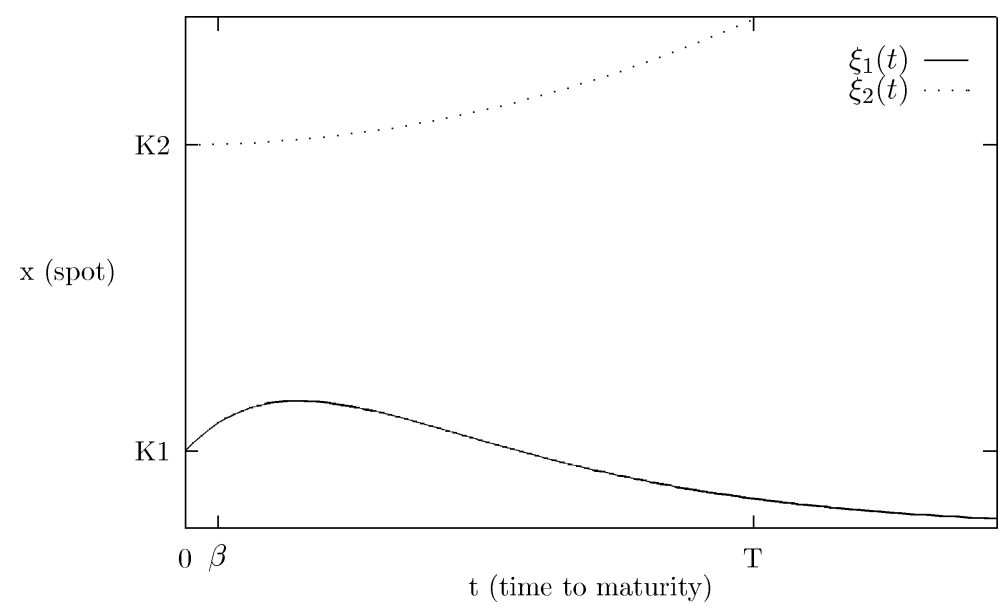

Fig. 1. 
Let $u>0$. The payoff $v_{\varphi}(u, x)$ satisfies (H0). Let

$$
\widehat{\varphi}_{u}(x)=\inf _{t \geqslant 0} v_{\varphi}(t+u, x)
$$

Since $(t, x) \rightarrow v_{\varphi}(t+u, x)$ is continuous and $t \rightarrow v_{\varphi}(t, x)$ is increasing for $t \geqslant t(x)$ where $t(x)$ is locally bounded (see Lemma 12), the function $\widehat{\varphi}_{u}(x)$ is continuous. According to Lemma 12, there exist $0<\beta<T<+\infty$ such that $t \rightarrow \xi_{1}(t)$ is strictly increasing on $[0, \beta]$ and strictly decreasing on $[T,+\infty)$. Concerning the existence of a continuous function $\widehat{t}_{u}$ such that $\widehat{\varphi}_{u}(x)=v_{\varphi}\left(\widehat{t}_{u}(x)+u, x\right)$ the situation depends on whether $u<\beta$ or $u \geqslant T$.

PROPOSITION 13.-

- If $u \geqslant T$, then $\widehat{\varphi}_{u}(x)=v_{\varphi}\left(\widehat{t}_{u}(x)+u, x\right)$ for the continuous function

$$
\widehat{t}_{u}(x)=1_{\left\{x \leqslant \xi_{1}(u)\right\}}\left(\xi_{1}^{-1}(x)-u\right)+1_{\left\{x \geqslant \xi_{2}(u)\right\}}\left(\xi_{2}^{-1}(x)-u\right),
$$

where $\xi_{1}^{-1}$ denotes the inverse of the restriction of $\xi_{1}$ to $[T,+\infty)$ and the price of the American option with payoff $\widehat{\varphi}_{u}$ is

$$
\begin{aligned}
v_{\widehat{\varphi}_{u}}^{a m}(t, x)= & v_{\varphi}\left(\left(t \vee \widehat{t}_{u}(x)\right)+u, x\right) \\
= & v_{\varphi}\left((t+u) \vee \xi_{1}^{-1}(x), x\right) 1_{\left\{x \leqslant \xi_{1}(u)\right\}}+v_{\varphi}(t+u, x) 1_{\left\{\xi_{1}(u)<x<\xi_{2}(u)\right\}} \\
& +v_{\varphi}\left((t+u) \vee \xi_{2}^{-1}(x), x\right) 1_{\left\{x \geqslant \xi_{2}(u)\right\}} .
\end{aligned}
$$

- If $u<\beta$, there is no continuous function $\widehat{t}_{u}$ such that $\widehat{\varphi}_{u}(x)=v_{\varphi}\left(\widehat{t}_{u}(x)+u, x\right)$. Moreover,

$$
\forall t>0, \forall x \in] \xi_{1}(t+u), \xi_{2}(t+u)\left[, \quad v_{\varphi}(t+u, x)>v_{\widehat{\varphi}_{u}}^{a m}(t, x)\right.
$$

Proof. - We first suppose that $u \geqslant T$. According to Lemma 12, $t \in[0,+\infty) \rightarrow$ $\xi_{1}(t+u)$ (respectively $\left.t \in[0,+\infty) \rightarrow \xi_{2}(t+u)\right)$ is decreasing (respectively increasing), and $\forall x \in] 0, \xi_{1}^{-1}(u)$ [ (respectively $\left.\forall x \in\right] \xi_{2}^{-1}(u),+\infty[) t \rightarrow v_{\varphi}(t+u, x)$ is decreasing on $\left[0, \xi_{1}^{-1}(x)-u\right]$ (respectively $\left[0, \xi_{2}^{-1}(x)-u\right]$ ) and increasing on $\left[\xi_{1}^{-1}(x)-u,+\infty[\right.$ (respectively $\left[\xi_{2}^{-1}(x)-u,+\infty[)\right.$. Moreover $\forall x \in\left[\xi_{1}(u), \xi_{2}(u)\right], t \rightarrow v_{\varphi}(t+u, x)$ is increasing. Hence $\widehat{\varphi}_{u}(x)=v_{\varphi}\left(\widehat{t}_{u}(x)+u, x\right)$ for the continuous function

$$
\widehat{t}_{u}(x)=1_{\left\{x \leqslant \xi_{1}(u)\right\}}\left(\xi_{1}^{-1}(x)-u\right)+1_{\left\{x \geqslant \xi_{2}(u)\right\}}\left(\xi_{2}^{-1}(x)-u\right),
$$

and we deduce the price of the American option with payoff $\widehat{\varphi}_{u}$ by Theorem 3 .

We turn to the case $u<\beta$. Let $F=\left\{(t, x): v_{\varphi}(t+u, x)=\widehat{\varphi}_{u}(x)\right\}$. According to Lemma $12, t \rightarrow \xi_{1}(t)$ is increasing on $[0, \beta]$. We deduce that $\left.\forall t \in\right] u, \beta\left[, v_{\varphi}\left(t, \xi_{1}(t)\right)>\right.$ $v_{\varphi}\left(u, \xi_{1}(t)\right)$ and $\left(t-u, \xi_{1}(t)\right) \notin F$. Hence

$$
\begin{aligned}
& F \subset F_{1} \cup F_{2} \quad \text { where } F_{1}=\left\{\left(t-u, \xi_{1}(t)\right), t \geqslant \beta\right\} \quad \text { and } \\
& F_{2}=\left\{\left(t-u, \xi_{2}(t)\right), t \geqslant u\right\} \cup\left\{(0, x), x \in\left[\xi_{1}(u), \xi_{2}(u)\right]\right\} .
\end{aligned}
$$


Let $\widehat{t}_{u}$ be such that $\forall x>0, \widehat{\varphi}_{u}(x)=v_{\varphi}\left(\widehat{t}_{u}(x)+u, x\right)$ i.e. $\left(\widehat{t}_{u}(x), x\right) \in F$. For $x$ small enough $\left(\widehat{t}_{u}(x), x\right) \in F_{1}$ and for $x$ big enough $\left(\widehat{t}_{u}(x), x\right) \in F_{2}$. Since $F_{1}$ and $F_{2}$ are not connected, the function $\widehat{t}_{u}$ is discontinuous.

Let $t>0$ and $x \in\left(\xi_{1}(t+u), \xi_{2}(t+u)\right)$. The positive continuous function

$$
w \in W \rightarrow \Phi(w)=\inf _{s \in[0, t]} \mathrm{e}^{-\rho s}\left(v_{\varphi}\left(t+u-s, x \mathrm{e}^{\sigma w_{s}+\left(\rho-\frac{\sigma^{2}}{2}\right) s}\right)-\widehat{\varphi}_{u}\left(x \mathrm{e}^{\sigma w_{s}+\left(\rho-\frac{\sigma^{2}}{2}\right) s}\right)\right),
$$

where $W=\{w \in C([0, t], \mathbb{R}), w(0)=0\}$, is not constantly equal to 0 . Indeed, when

$$
\begin{aligned}
& \forall s \leqslant 0 \vee(t+u-\beta), \quad \xi_{1}(t+u-s)<x \mathrm{e}^{\sigma w_{s}+\left(\rho-\frac{\sigma^{2}}{2}\right) s}, \\
& x \mathrm{e}^{\sigma w_{t}+\left(\rho-\frac{\sigma^{2}}{2}\right) t}<\xi_{1}(u), \\
& \text { and } \forall s \leqslant t, \quad x \mathrm{e}^{\sigma w_{s}+\left(\rho-\frac{\sigma^{2}}{2}\right) s}<\xi_{2}(t+u-s),
\end{aligned}
$$

then $\forall s \in[0, t],\left(t-s, x \mathrm{e}^{\sigma w_{s}+\left(\rho-\frac{\sigma^{2}}{2}\right) s}\right) \notin F$ and $\Phi(w)>0$. As the support of the Wiener measure is $W, \mathbb{E}\left[\Phi\left(\left(B_{s}\right)_{s \leqslant t}\right)\right]>0$. Let $\tau$ be a stopping time smaller than $t$. Then

$$
\begin{aligned}
v_{\varphi}(t+u, x) & =\mathbb{E}\left[\mathrm{e}^{-\rho \tau} v_{\varphi}\left(t+u-\tau, X_{\tau}^{x}\right)\right] \\
& \geqslant \mathbb{E}\left[\mathrm{e}^{-\rho \tau} \widehat{\varphi}_{u}\left(X_{\tau}^{x}\right)\right]+\mathbb{E}\left[\Phi\left(\left(B_{s}\right)_{s \leqslant t}\right)\right] .
\end{aligned}
$$

Since $\tau$ is arbitrary, we conclude that $v_{\varphi}(t+u, x)-v_{\widehat{\varphi}_{u}^{a m}}^{a m}(t, x) \geqslant \mathbb{E}\left[\Phi\left(\left(B_{s}\right)_{s \leqslant t}\right)\right]>0$.

Remark 14. -

(1) For any $x>0, t \rightarrow v_{\varphi}(t+u, x)$ is continuous and increasing for $t$ big enough. Hence $\tilde{t}_{u}(x)=\sup \left\{t: v_{\varphi}(t+u, x)=\widehat{\varphi}_{u}(x)\right\}$ is finite. When $u<\beta, \forall t \leqslant \tilde{t}_{u}(x)$,

$$
\begin{aligned}
& \widehat{\varphi}_{u}(x) \leqslant v_{\widehat{\varphi}_{u}}^{a m}(t, x) \leqslant v_{\widehat{\varphi}_{u}}^{a m}\left(\tilde{t}_{u}(x), x\right) \leqslant v_{\varphi}\left(\tilde{t}_{u}(x)+u, x\right), \\
& \text { i.e. } \quad v_{\widehat{\varphi}_{u}}^{a m}(t, x)=\widehat{\varphi}_{u}(x),
\end{aligned}
$$

but $\exists T(x)$ such that for $t \geqslant T(x), x \in] \xi_{1}(t+u), \xi_{2}(t+u)[$ and we cannot deduce $v_{\hat{\varphi}_{u}}^{a m}(t, x)$ from the price of the European option with payoff $\varphi$.

(2) Let $u<\beta$ and $x^{*}=\sup \left\{x:(t, x) \in F_{1} \cap F\right\}$ where $F, F_{1}$ are defined in the previous proof. Since $F_{1}$ and $F$ are closed and $\lim _{t \rightarrow+\infty} \xi_{1}(t)=0, \exists t^{*} \geqslant \beta-u$ such that $\left(t^{*}, x^{*}\right) \in F_{1} \cap F$ i.e. $v_{\varphi}\left(t^{*}+u, x^{*}\right)=\widehat{\varphi}_{u}\left(x^{*}\right)$. Since $x^{*}=\sup \{x:(t, x) \in$ $\left.\left.\left.F_{1} \cap F\right\}, \forall x \in\right] x^{*}, \xi_{2}(u)\right], v_{\varphi}(u, x)=\widehat{\varphi}_{u}(x)$ and by continuity, $v_{\varphi}\left(u, x^{*}\right)=\widehat{\varphi}_{u}\left(x^{*}\right)$. Hence $\left\{t \geqslant 0, v_{\varphi}\left(t+u, x^{*}\right)=\widehat{\varphi}_{u}\left(x^{*}\right)\right\}$ contains at least two elements which is not surprising with regard to Remark 4.

\section{Analyticity and some consequences}

In this section we give some properties of the map $\varphi \rightarrow \widehat{\varphi}$ which are consequences of the following analyticity of $v_{\varphi}$ in the pair $(t, x)$ :

Proposition 15. - The function $v_{\varphi}(t, x)$ is analytic in $] 0, \infty\left[\times \mathbb{R}_{+}^{*}\right.$.

This is a consequence of the same property for the solution of the standard heat equation (which does not seem to be universally known in fact but can be shown by a direct estimation of the derivatives of the solution). 


\subsection{One-to-one property}

Let us study now the injectivity of $\varphi \rightarrow \widehat{\varphi}$. Obviously if $\varphi_{1}$ is an invariant function (e.g. the nil function), then $\widehat{\varphi}_{1}=\varphi_{1}$ and there are plenty of other functions $\varphi$ such that $\widehat{\varphi}=\widehat{\varphi}_{1}$, for instance $\varphi=\varphi_{1}+\phi$ where $\phi$ is a non-negative continuous function satisfying $\lim _{x \rightarrow 0} \frac{\phi(x)}{x+x^{-\alpha}}=\lim _{x \rightarrow+\infty} \frac{\phi(x)}{x+x^{-\alpha}}=0$. The same phenomenon occurs if $\widehat{t}(x)=$ $\infty$ everywhere. Therefore the following one-to-one statement is optimal:

PROPOSITION 16. - Let $\varphi_{1}$ and $\varphi_{2}$ satisfy the assumptions of Theorem 3 and assume that $\varphi_{1}$ is not an invariant function and that there is a value $x_{0}$ such that $\widehat{t}_{1}\left(x_{0}\right)<\infty$. Then

$$
\widehat{\varphi}_{1}=\widehat{\varphi}_{2} \Rightarrow \varphi_{1}=\varphi_{2}
$$

Proof. - For any $t_{0}>\widehat{t}_{1}\left(x_{0}\right)$ there is an $\varepsilon>0$ small enough such that the ball $B$ centered at $\left(t_{0}, x_{0}\right)$ with radius $\varepsilon$ lies in the Continuation region of $\hat{\varphi}_{1}$. In particular $v_{\widehat{\varphi}_{1}}^{a m}(t, x)=v_{\varphi_{1}}(t, x)$ on $B$ and $(t, x) \mapsto v_{\widehat{\varphi}_{1}}^{a m}(t, x)$ is analytic on $B$. Now $v_{\widehat{\varphi}_{1}}^{a m}(t, x)=$ $v_{\widehat{\varphi}_{2}}^{a m}(t, x)$, therefore $v_{\widehat{\varphi}_{2}}^{a m}(t, x)$ is analytic on $B$. If $\widehat{t}_{2}\left(x_{0}\right)=+\infty$ then by taking $\varepsilon$ small enough we can assume $v_{\hat{\varphi}_{2}}^{a m}(t, x)=\widehat{\varphi}_{2}(x)$ on $B$. Therefore $\partial_{t} v_{\varphi_{1}}(t, x)=0$ on $B$ and by the analyticity of $v_{\varphi_{1}}, \partial_{t} v_{\varphi_{1}}(t, x)=0$ everywhere, which gives that $\varphi_{1}$ is an invariant function. Since this case is ruled out by assumption, $\widehat{t_{2}}\left(x_{0}\right)<\infty$. Now on the right of $\widehat{t}_{1}\left(x_{0}\right) \vee \widehat{t}_{2}\left(x_{0}\right), v_{\varphi_{1}}$ and $v_{\varphi_{2}}$ match on some small enough ball, therefore everywhere, which gives $\varphi_{1}=\varphi_{2}$ by the continuity of $t \mapsto v_{\varphi_{i}}(t, x)$ at $t=0$.

\subsection{On the range of $\varphi \rightarrow \widehat{\varphi}$}

PROPOSITION 17. - Let $\varphi$ satisfy the assumptions of Theorem 3 and assume that $\varphi$ is not an invariant function and that $0<\widehat{t}\left(x_{0}\right)<\infty$ for some point $x_{0}$. Then there is an open dense subset of $\widehat{t}^{-1}\left(\mathbb{R}_{+}^{*}\right)$ on which $\widehat{t}$, and therefore $\widehat{\varphi}$, is analytic.

Proof. - First note that by composition $\widehat{\varphi}(x)=v_{\varphi}(\widehat{t}(x), x)$ is analytic as soon as $\widehat{t}$ is. Pick up some point $x_{1}$ such that $0<\widehat{t}\left(x_{1}\right)<\infty$. Since $v_{\varphi}$ is analytic, by the implicit function theorem the equation $\partial_{t} v_{\varphi}(t, x)=0$ on a small enough neighborhood $V$ of $\left(\widehat{t}\left(x_{1}\right), x_{1}\right)$ defines an analytic curve $x \mapsto a(x)$ as soon as $\partial_{t^{2}}^{2} v_{\varphi}\left(\widehat{t}\left(x_{1}\right), x_{1}\right) \neq 0$. Now by the continuity of $\widehat{t}, \partial_{t} v_{\varphi}(\widehat{t}(x), x)=0$ in $V$ so that $a \equiv \widehat{t}$ on a neighborhood of $x_{1}$.

Unfortunately it is not granted that there exists $x_{1}$ such that $\partial_{t^{2}}^{2} v_{\varphi}\left(\widehat{t}\left(x_{1}\right), x_{1}\right) \neq 0$.

Let us first remark that there is some point $x_{2}$ in $\widehat{t}^{-1}\left(\mathbb{R}_{+}^{*}\right)$ such that $t \mapsto v_{\varphi}\left(t, x_{2}\right)$ is not constant: otherwise $\partial_{t} v_{\varphi}(t, x)=0$ would hold on some non-empty open set and therefore everywhere, thus $\varphi$ would be an invariant function. Moreover the set of such points is dense in $\widehat{t}^{-1}\left(\mathbb{R}_{+}^{*}\right)$. Let $q=\inf \left\{n>0, \partial_{t^{n}}^{n} v_{\varphi}\left(\widehat{t}\left(x_{2}\right), x_{2}\right) \neq 0\right\}$. Then $q$ is finite. If $q=2$ we are over. Otherwise notice first that the equation $\partial_{t q-1}^{q-1} v_{\varphi}(t, x)=0$ on a small enough neighborhood $V$ of $\left(\widehat{t}\left(x_{2}\right), x_{2}\right)$ defines an analytic curve $x \mapsto b(x)$. Consider then the quantity $q(x)=\inf \left\{n>0, \partial_{t^{n}}^{n} v_{\varphi}(\widehat{t}(x), x) \neq 0\right\}$ on a neighborhood of $x_{2}$. By the analyticity of $v_{\varphi}, q(x) \leqslant q\left(x_{2}\right)$ on a sufficiently small neighborhood $W$ of $x_{2}$. Either $q(x) \equiv q\left(x_{2}\right)$, in which case $\partial_{t^{q-1}}^{q-1} v_{\varphi}(\widehat{t}(x), x)=0$ on $W$, therefore $\widehat{t} \equiv b$ is analytic on $W$, otherwise there is some point $x_{3}$ in $W$ such that $0<\widehat{t}\left(x_{3}\right)<\infty$ and $q\left(x_{3}\right)<q\left(x_{2}\right)$. 
By induction we thus either stop and get an analytic curve or reach the level $q=2$ at some point. The proof is complete.

This proposition gives a first characterization statement about the functions $\widehat{\varphi}$ :

COROLlaRY 18. - Let $\varphi$ satisfy the assumptions of Theorem 3. Then $\widehat{\varphi}$ is either $\mathcal{A}$ superharmonic (i.e. $\mathcal{A} \widehat{\varphi} \geqslant 0$ in a weak sense) or $\mathcal{A}$-subharmonic (i.e. $\mathcal{A} \widehat{\varphi} \leqslant 0$ in a weak sense) or analytic on a non-empty open subset of $\mathbb{R}_{+}^{*}$. In particular $\varphi \rightarrow \widehat{\varphi}$ is not onto on the space of functions satisfying (H0).

Proof. - The first case corresponds to $\widehat{t}=0$ everywhere, the second one to the case $\widehat{t}=+\infty$ everywhere $\left(\widehat{\varphi}(x)=\liminf _{t \rightarrow+\infty} v_{\varphi}(t, x)\right.$ is then an $\mathcal{A}$-subharmonic function by Fatou's lemma) and the last one to the previous proposition.

\section{Conclusion}

In this paper, for a fairly general class of payoffs $\varphi$, we deduce from the European price $v_{\varphi}(t, x)$ the American price of the claim with payoff $\hat{\varphi}(x)=\inf _{t \geqslant 0} v_{\varphi}(t, x)$. We give examples of explicit computations. The characterization of the payoffs $\widehat{\varphi}$ obtained in this way remains an open question. A work devoted to design new approximations of the American Put price relying on our approach is in progress.

\section{Acknowledgements}

We thank Régis Monneau (CERMICS) for numerous stimulating and fruitful discussions.

\section{REFERENCES}

[1] Crank J., Free and Moving Boundary Problems, Oxford University Press, 1984.

[2] Martini C., The UVM model and American options, Rapport de Recherche no 3697, INRIA, 1999.

[3] Musiela M., Rutkowski M., Martingale Methods In Financial Modelling, Springer, 1998.

[4] Revuz D., Yor M., Continuous Martingales and Brownian Motion, Springer, 1991. 